\title{
COVID-19 and physician ethics
}

\author{
Gilbert Berdine
}

CoviD-19 has had a profound impact on the health care world. Although we know a lot more about this disease than we did in March, there is still a lot we do not know. While procedures play an increased role in the physician-patient relationship than they did 50 years ago, the role of physicians remains one of giving expert advice to patients about how best to improve their health. Questions (and the answers to questions) about whether symptoms imply COVID-19 disease, whether COVID-19 requires medical intervention, and, if intervention is deemed necessary, what intervention is best have become a very important part of the interactions between physicians and patients. Physicians are faced with decisions about diagnosis and decisions about therapy. There is necessarily uncertainty involved with these decisions. Physicians give advice. In some cases, the advice is accepted, but in other cases it is not. This discussion will be about the ethics of handling these conflicts between physician and patient.

\section{Patient Requests a therapy that a PHYSICIAN CONSIDERS TO BE DANGEROUS}

As of this time, I do not believe that any of the controversial therapies for COVID-19 have been shown to be harmful, so this will be a contrived hypothetical case. Suppose a patient with COVID-19 has developed acute respiratory distress syndrome (ARDS) with bilateral pulmonary infiltrates on chest imaging and severe hypoxemia. The patient requires mechanical ventilation. Suppose this patient decides that the only way to survive this illness is bilateral lung resection and extra corporeal membrane oxygenation (ECMO) pending double lung transplant. Is the physician required to grant this request? The answer is clearly no. If the patient is young enough to be a viable candidate for

Corresponding author: Gilbert Berdine

Contact Information: Gilbert.Berdine@ttuhsc.edu

DOI: $10.12746 /$ swrccc.v9i37.791 lung transplantation, then the expected recovery from the COVID-19 would exceed the expected long-term survival from lung transplant. If the patient is old enough for a high expected mortality from COVID-19, then the patient is too old for lung transplant. The correct decision by the physician would be to treat the COVID-19 with supportive care, dismiss the thought of lung resection out of hand, and only consider ECMO or lung transplant should the patient meet the usual criteria for these interventions following usual care. If the patient demands lung resection, the physician would be correct to refuse. The patient would be free to find another physician willing to grant the request, but neither the treating physician nor the treating hospital would be obligated to find this other physician for the patient.

\section{PATIENT REQUESTS A THERAPY THAT PHYSICIAN DEEMS NEITHER HELPFUL NOR HARMFUL}

There are a number of examples in this category. Hydroxychloroquine and convalescent serum are two examples. Both treatments have advocates who swear by anecdotal evidence, but randomized controlled trials fail to confirm efficacy. This is a dilemma for the physician. The dilemma is created by government licensure of physicians as gatekeepers preventing patients from receiving therapy without permission. In the absence of this licensure, physicians would be free to advise against ineffective therapy and patients would be free to ignore this advice. If the physician does not permit the patient access to the treatment, the patient will believe that whatever the outcome was worse than what would have occurred had the treatment been permitted. For simple low-cost remedies with few or no side effects, like azithromycin, the physician will most likely grant the request. For expensive treatments, the physician may be pressured by the thirdparty payer to refuse access for cost reasons.

My own approach to these problems considers only the risk vs. benefit assessment and ignores costs to third parties. If the risk vs. benefit is heavily in favor of 
risk, then I advise against the treatment. If the patient does not agree with my advice, then the patient must find another physician to get the desired treatment. If the risk vs. benefit is roughly equal and only slightly in favor of risk, then I advise against the therapy. If the patient does not agree with my advice, I document in the medical record the discussion of risk vs. benefit, further document that the fully informed patient chose risk, and grant access to the therapy. I would much prefer the patient be able to get the therapy without my approval, so I make sure that my dissent is well recorded.

I consider myself to have a contractual relationship with the patient. I am the advocate for the patient. My decisions are based on risk vs. benefit to the patient rather than to the hospital or some third-party payer. Consider the example of some very expensive therapy. The hospital has the option of removing the therapy from the formulary or restricting access by some third-party gate keeper. Examples include new antibiotics that cannot be used without approval from an infectious disease expert. Third party payers including both private insurance companies and government entities like Medicare or Medicaid deny payment for certain services all the time.

\section{Patient declines a therapy that Physician DEEMS HELPFUL}

This happens all the time. Normally physicians respect patient autonomy. An example would be a patient with COVID-19 develops worsening respiratory failure with hypoxemia. The physician recommends endotracheal intubation and mechanical ventilation. The patient declines. The clearly correct answer is to respect the patient's wish.

Keywords: COVID-19, risk-benefit, ethics, autonomy

Article citation: Berdine G. COVID-19 and physician ethic. The Southwest Respiratory and Critical Care Chronicles 2021;9(37):82-83

From: Department of Internal Medicine, Texas Tech University Health Sciences Center, Lubbock, Texas

Submitted: $11 / 27 / 2020$

Accepted: $12 / 13 / 2020$

Conflicts of interest: none

This work is licensed under a Creative Commons Attribution-ShareAlike 4.0 International License. 